\title{
Effect of non-Gaussian noise sources in a noise-induced transition
}

\author{
Horacio S. Wio*, Raúl Toral \\ Departament de Física, Universitat de les Illes Balears, Instituto Mediterráneo de Estudios Avanzados, \\ IMEDEA (CSIC-UIB), Ed. Mateu Orfila, Campus UIB, E-07122 Palma de Mallorca, Spain
}

\begin{abstract}
Here we study a noise-induced transition when the system is driven by a noise source taken as colored and non-Gaussian. We show-using both, a theoretical approximation and numerical simulations-that there is a shift of the transition as the noise departs from the Gaussian behavior. Also, we confirm the reentrance effect found for colored Gaussian noise and show the behavior of the transition line in the phase-like diagram as the noise departs from Gaussianity in the large correlation time limit.

(C) 2004 Elsevier B.V. All rights reserved.
\end{abstract}

PACS: 05.40.-a; 02.50.Ey; 05.10.Ln

Keywords: Non-Gaussian noise; Noise-induced transition; Non-extensivity

\section{Introduction}

During the last three decades a wealth of research results on fluctuations or noise have led us to the recognition that in many situations noise can actually play a constructive role that induces new ordering phenomena. Some examples are stochastic resonance in zero-dimensional and extended systems [1-3], noise-induced transitions [4], noise-induced phase transitions [5,6], noise-induced transport [7-10], noise sustained patterns [11], etc.

Most of the studies on the noise-induced phenomena indicated above assume that the noise source has a Gaussian distribution (either white or colored). In addition to the intrinsic interest in the study of non-Gaussian noises, there are some experimental evidences, particularly in sensory and biological systems [12], indicating that at least in some of these phenomena the noise sources could be non-Gaussian. The use of non-Gaussian noises in studies on noise-induced phenomena is scarce mainly due to the mathematical difficulties [13]. This is in contrast with the existence of some analytical tools when working with Gaussian (particularly white) noises.

Here we present some results on one of those noise-induced phenomena when driven by a noise source taken as colored and non-Gaussian. It corresponds to a noise-induced transition like those discussed in [4]. The problem we

\footnotetext{
* Corresponding author. Permanent address: Centro Atómico Bariloche (CNEA), Instituto Balseiro (CNEA and UNCuyo), 8400 San Carlos de Bariloche, Argentina.

E-mail addresses: wio@imedea.uib.es (H.S.Wio), raul@imedea.uib.es (R. Toral).
} 
discuss here corresponds the so-called genetic model which follows from a particular biological modeling or from a chemical reaction [4,14]. In Ref. [15] the effect of a colored Gaussian (Ornstein-Uhlenbeck) noise on that model was analyzed in detail and a novel reentrance phenomenon in the phase diagram was found. In order to study the effect that the non-Gaussian nature of the noise can have in the transition, we have used here a particular distribution [16] whose departure from the Gaussian behavior is governed by a parameter $q$ : while $q=1$ corresponds to Gaussian noise, the value $q>1$ produces a long tail distribution and $q<1$ gives a cut-off distribution. Such a distribution is based on the generalized thermostatistics proposed by Tsallis [17] which has been widely applied to a variety of physical systems (for a state of the art see [18]). As discussed for other noise-induced phenomena in [19], we have found that the departure from the Gaussian distribution for the noise produces a strong effect. In this case such an effect corresponds to an anticipation of the transition when the distribution has long tails, and to a retardation when it is cut-off. This effect could have interesting consequences in the case of noise-induced-phase transitions [5,6].

In the next section we briefly discuss the form and properties of the non-Gaussian noise source. After that, we present the model we analyze here and a simple analytical approximation to treat the non-Gaussian noise that allows us to determine some of the general features of the phase diagram. We continue discussing numerical simulations that support and extend the theoretical results. In the last section we draw some conclusions.

\section{Non-Gaussian noise and its properties}

We start considering the following general form of a Langevin equation:

$$
\dot{x}=f(x)+g(x) \eta(t) .
$$

As described in Section 1, we assume that the noise term $\eta(t)$ has a non-Gaussian distribution. More precisely, we consider that $\eta(t)$ is a Markovian process generated as the solution of the following Langevin equation [16]:

$$
\dot{\eta}=-\frac{1}{\tau} \frac{\mathrm{d}}{\mathrm{d} \eta} V_{q}(\eta)+\frac{\sqrt{2 D}}{\tau} \xi(t)
$$

being $\xi(t)$ a standard Gaussian white noise of zero mean and correlation $\left\langle\xi(t) \xi\left(t^{\prime}\right)\right\rangle=\delta\left(t-t^{\prime}\right)$, and

$$
V_{q}(\eta)=\frac{D}{\tau(q-1)} \ln \left[1+\frac{\tau}{D}(q-1) \frac{\eta^{2}}{2}\right] .
$$

Although we believe that our results are quite general, such a particular form for the noise $\eta(t)$ allows us to easily control the departure from the Gaussian behavior by changing a single parameter $q$. $D$ and $\tau$ are noise parameters related to the noise intensity and the correlation time, as we now detail. The stationary properties of the noise $\eta$, including the time correlation function, have been studied in [20] and here we summarize the main results. The stationary probability distribution is given by

$$
P_{q}^{\mathrm{st}}(\eta)=\frac{1}{Z_{q}}\left[1+\frac{\tau}{D}(q-1) \frac{\eta^{2}}{2}\right]^{-1 /(q-1)},
$$

where $Z_{q}$ is the normalization factor. This distribution can be normalized only for $q<3$. The first moment, $\langle\eta\rangle=0$, is always equal to zero, and the second moment,

$$
\left\langle\eta^{2}\right\rangle=\frac{2 D}{\tau(5-3 q)},
$$

is finite only for $q<5 / 3$. Furthermore, for $q<1$, the distribution has a cut-off and it is only defined for $|\eta|<\eta_{\mathrm{c}} \equiv$ $\sqrt{2 D / \tau(1-q)}$. Finally, the correlation time $\tau_{q}$ of the stationary regime of the process $\eta(t)$ diverges near $q=5 / 3$ 
and it can be approximated over the whole range of values of $q$ as $\tau_{q} \approx 2 \tau /(5-3 q)$. Clearly, when $q \rightarrow 1$ we recover the limit of $\eta$ being a Gaussian colored noise, namely the Ornstein-Uhlenbeck process, $\xi_{\text {ou }}(t)$, with correlations $\left\langle\xi_{\text {ou }}(t) \xi_{\text {ou }}\left(t^{\prime}\right)\right\rangle=(D / \tau) \mathrm{e}^{-\left|t-t^{\prime}\right| / \tau}$ and probability distribution $P^{\text {st }}\left(\xi_{\text {ou }}\right)=Z^{-1} \mathrm{e}^{-(\tau / 2 D) \xi_{\text {ou }}^{2}}$.

In Ref. [20], an effective Markovian approximation to the process $\eta$ via a path integral procedure was obtained, that is analogous to the "unified colored noise approximation" obtained in [21] for the case of Ornstein-Uhlenbeck noise. Such an approximation allowed us to get quasi-analytical results for the mean-first-passage time or transition rate. These results and its dependence on the different parameters in the case of a double well potential were compared with extensive numerical simulations with excellent agreement. That approximation was also exploited to study stochastic resonance with theoretical results [22] that are in very good agreement with experimental ones [23].

\section{3. $q \approx 1$ approximation for the non-Gaussian noise}

The "effective Markovian approximation" introduced in Ref. [20] and indicated above has been shown to have some drawbacks. As discussed in [20], such an approximation renders a non-normalizable stationary probability distribution function, $P_{q}^{\text {st }}(\eta)$. This seems to be a general characteristic of such an approximation that, at least in the case discussed in [20], was solved introducing an adequate and controlled cut-off. However, it is not clear that such a methodology could be always used, and particularly for the models we can analyze in relation to noise-induced transitions. Hence, for the kind of models we use in order to study the effect of non-Gaussian noises on a noise-induced transition we have resorted to a more simple, albeit physically reasonable, approximation. Our aim is to analytically study the region $|q-1| \ll 1$ (both for $q<1$ and $q>1$ ), with the idea that in such a region the non-Gaussian noise will only slightly depart from the Gaussian behavior, but will show some of the main trends of the $q \neq 1$ region. Hence in Eq. (2) we adopt

$$
\frac{1}{\tau} \frac{\partial V_{q}(\eta)}{\partial \eta}=\frac{\eta}{\tau}\left[1+\frac{\tau}{D}(q-1) \frac{\eta^{2}}{2}\right]^{-1} \approx \frac{\eta}{\tau}\left[1+\frac{\tau}{D}(q-1) \frac{\left\langle\eta^{2}\right\rangle}{2}\right]^{-1} \equiv a(\tau) \eta
$$

with

$$
\tau_{\mathrm{eff}}=\frac{2(2-q)}{5-3 q} \tau
$$

This implies a renormalized (Gaussian) Ornstein-Uhlenbeck noise where the effective correlation time is indicated above (Eq. (7)), and the associated effective noise intensity is $D_{\text {eff }}=(2(2-1) /(5-3 q))^{2} D$. Clearly, for $q \rightarrow 1$, we have $\tau_{\text {eff }} \rightarrow \tau$ and $D_{\text {eff }} \rightarrow D$, as could be expected. Using this form of the noise we proceed to study the problem in Eq. (1) in the neighborhood of $q \approx 1$.

The genetic model is described by the following equation $[4,14,15]$ :

$$
\dot{x}=\frac{1}{2}-x+\lambda x(1-x)+x(1-x) \eta(t),
$$

and, according to the discussion above, we consider in this work that $\eta(t)$ is a non-Gaussian noise described by Eqs. (2) and (3).

The analytical treatment simplifies if we make the change of variables

$$
y=\ln \left(\frac{x}{1-x}\right)
$$

so that the original multiplicative stochastic differential equation (8) transforms into a new one but now with an additive noise 


$$
\dot{y}=-\sinh (y)+\lambda+\eta(t) .
$$

From now on, as in Ref. [15], and in order to simplify the algebra, in what follows we adopt $\lambda=0$.

The use of the (Gaussian-like) approximation Eq. (6) (that we reiterate could be valid only for $|q-1| \ll 1$ ) together with the "unified colored noise approximation" [21], yields

$$
\dot{y} \approx f(y) \frac{1}{\left[1-f^{\prime}(y) / a(\tau)\right]}+\frac{1}{\tau a(\tau)} \frac{1}{\left[1-f^{\prime}(y) / a(\tau)\right]} \xi(t)
$$

with $f(y)=-\sinh (y)$. This result can be obtained in two ways: (a) Applying a direct adiabatic-like elimination procedure to the Langevin system given by Eq. (10) and Eqs. (2) and (6) [21]. That is, taking the derivative of Eq. (10) with respect to $t$ and setting $\ddot{y}=0$. (b) A formal one, through the application of the path-integral formalism to the indicated non-Markovian Langevin equations, supplemented with a consistent Markovian approximation scheme [24].

Since, by the use of these "drastic" approximations, the problem has been reduced to Eq. (11) which contains a white, Gaussian, noise, it is straightforward now, using standard techniques, to obtain the stationary probability density function. For the original variable $x$, it reads

$$
P_{\mathrm{st}}(x)=N \exp [F(x)],
$$

where $N$ is a normalization constant and

$$
\begin{aligned}
F(x)= & -\frac{1}{8 D}\left(\frac{5-3 q}{2-q}\right)^{2} \frac{1-2 x+2 x^{2}}{x(1-x)}\left(1+\frac{\tau(2-q)\left(1-2 x+2 x^{2}\right)}{2(5-3 q) x(1-x)}\right) \\
& +\ln \left[1+\frac{\tau(2-q)\left(1-2 x+2 x^{2}\right)}{(5-3 q) x(1-x)}\right]-\ln [x(1-x)] .
\end{aligned}
$$

A noise-induced transition [4] appears when this density function changes from being unimodal to bimodal. More specifically, the point $x=1 / 2$ changes from being a maximum to being a minimum. The condition of extremum at $x=1 / 2$ and the condition for finding the boundary separating the regions where $x=1 / 2$ is a maximum or a minimum yield

$$
D(\tau)=\frac{1}{2}\left(\frac{5-3 q}{2-q}\right)^{2}\left(1+\frac{2-q}{5-3 q} 2 \tau\right)^{2}\left(1+\frac{2-q}{5-3 q} 6 \tau\right)^{-1},
$$

such that for a fixed $\tau$, the distribution is bimodal for $D>D(\tau)$ and it is unimodal for $D<D(\tau)$. This last expression can be written in terms of $D_{\text {eff }}$ and $\tau_{\text {eff }}$ as

$$
D_{\text {eff }}\left(\tau_{\text {eff }}\right)=2\left(1+\tau_{\text {eff }}\right)^{2}\left(1+3 \tau_{\text {eff }}\right)^{-1},
$$

that has the same form found in [15] for the case $q=1$. Clearly, in the limit $q \rightarrow 1$, this expression reduces to the indicated result, i.e.: $D(\tau)=2[1+\tau]^{2}[1+3 \tau]^{-1}$.

Fig. 1 plots the $D(\tau)$ boundary (the "transition line") for different values of $q$ in the $(D, \tau)$ plane. As indicated above, the approximation we have just described is only strictly valid for values of $q$ such that $|q-1| \ll 1$. However, and only in order to make more apparent the main trends predicted by our "drastic" approximation for the non-Gaussian case, we have plotted Eq. (14) for values of $q$ well departed from $q=1$. Several features can be noticed in this figure. It is clear that a minimum value of the noise intensity $D$ is always needed to induce the transition. The dependence with the parameter $q$ appears as an advancement of the transition to smaller values of $D$ when $q>1$ and a retardation when $q<1$. We believe that this will be a general behavior for long tail (power-law) and cut-off noise distributions, respectively. A closer look shows the persistence of the reentrance effect previously 


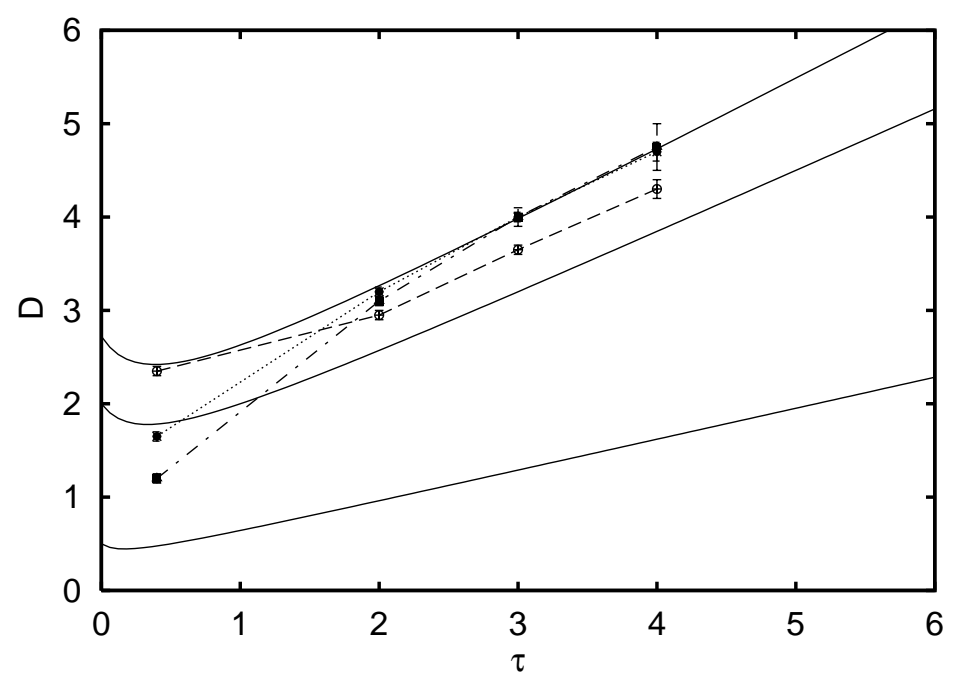

Fig. 1. Phase diagram $D$ vs. $\tau$ for different values of $q$. Probability distributions are bimodal for points above the curves and unimodal below. Solid lines are the theoretical predictions, as given by Eq. (14) for $q=0.5, q=1, q=1.5$ (from top to bottom). The numerical results are indicated by symbols joined by a dashed line for $q=0.5$, a dotted line for $q=1$, and a dash-dotted for $q=1.5$. It is clear the reentrance effect and the shift of the transition line.

found in [15]: for some fixed values of the noise intensity $D$ it is possible to obtain a transition, first from unimodal to bimodal and then back to unimodal, just by varying the time correlation $\tau$ of the noise.

These main features, as obtained from our very simplifying assumptions have been supported by the results from numerical simulations of the complete (that is for all values of $q$ ) set of Eqs. (2), (3) and (8) (details of the numerical integration scheme are given in Appendix A). In Fig. 2 we show that the probability distribution can

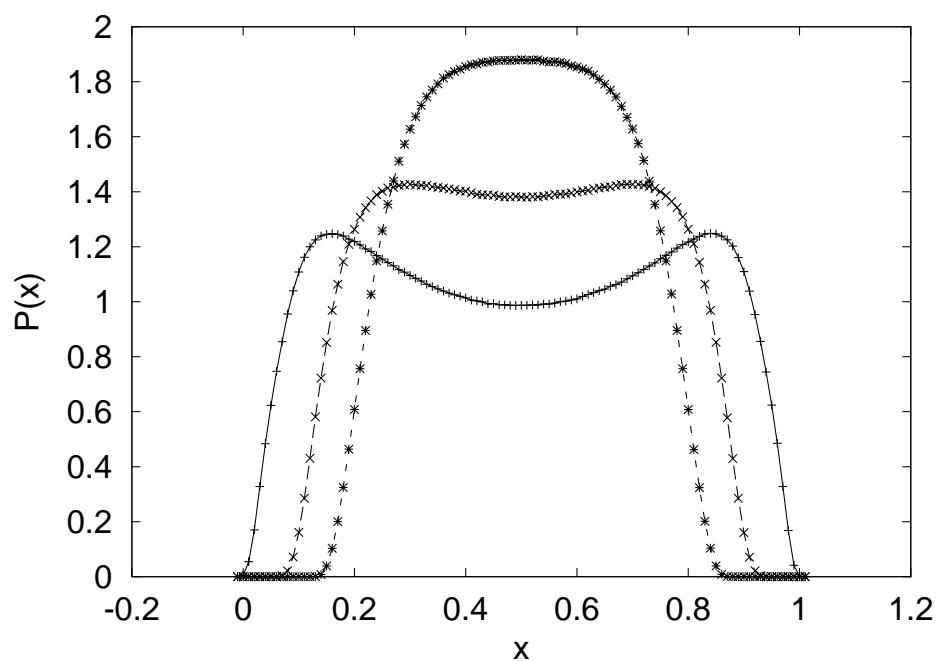

Fig. 2. Simulation results for the stationary probability distribution $P_{\mathrm{st}}(x)$ for fixed values $D=2, \tau=0.4$ and different values of $q$ : $q=0.5$ $(*), q=1(\times), q=1.5(+)$. It is clear that it is possible to induce a transition from unimodal to bimodal just by changing the parameter $q$, measuring the departure from the Gaussian distribution for the noise source. 


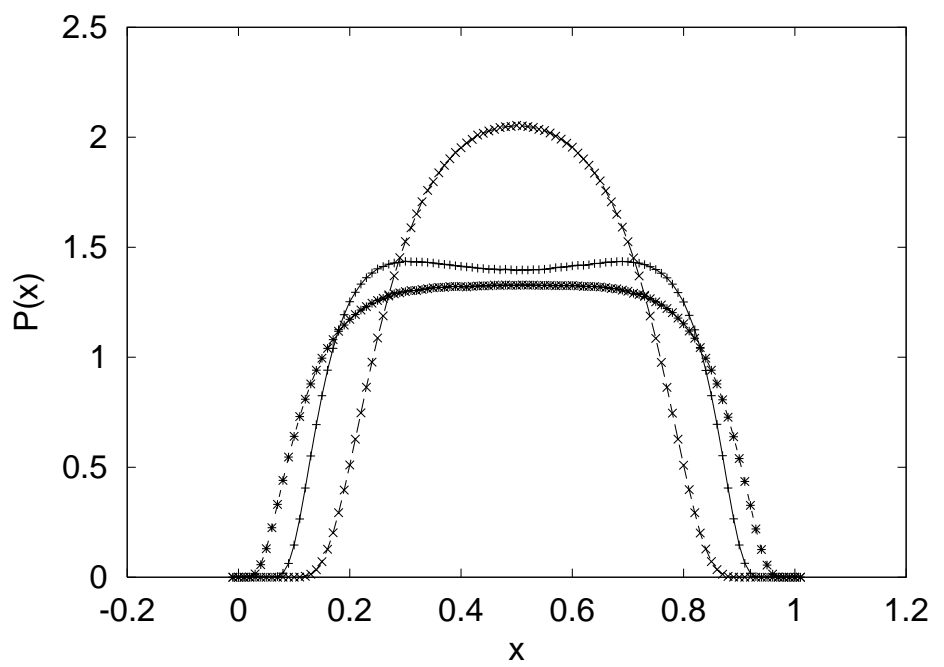

Fig. 3. Simulation results for the stationary probability distribution $P_{\mathrm{st}}(x)$ for $q=1$ (Gaussian case) for fixed $D=1.95$, and different values of $\tau: \tau=0 .(\times), \tau=0.4(+), \tau=1.0(*)$. The reentrance effect indicated in the phase diagram $D$ vs. $\tau$ is apparent.

switch from unimodal to bimodal just by increasing the parameter $q$, for fixed values of $D$ and $\tau$. The reentrance effect is illustrated, for $q=1$ in Fig. 3.

The numerical simulations show that there is a crossing of the transition lines for $q<1$ and $q \geq 1$, an aspect which is not predicted at all by the approximated theoretical results. Another aspect observed in Fig. 1 is that for large $\tau$, the theoretically predicted transition line, has a smaller linear slope for increasing values of $q$. In fact, according to Eq. (14) we have $D \sim((5-3 q) / 3(2-q)) \tau$ in that limit. There seems to be not such a strong $q$ dependence of the slope of the curves for large $\tau$ in the numerical results. Otherwise, the numerical and theoretical results are in good qualitative agreement.

\section{Final remarks}

We have presented some preliminary results on a noise-induced phenomenon driven by a colored and non-Gaussian noise source that was generated by a $q$-distribution. The phenomenon studied here is a noise-induced transition like those discussed in [4], and corresponds to the so-called genetic model. We have resorted to a simple approximation — that avoids the difficulties of the effective Markovian approximation in [20]—introduced with the idea that for $|q-1| \ll 1$, the non-Gaussian noise will only slightly depart from the Gaussian one, and its effect being only a correction to the Gaussian behavior. The resulting Eq. (14) shows some of the correct tendencies with $q>1$ or $q<1$, although some features observed in the simulations (crossing of the transition lines and slight dependence of the linear slope for large $\tau$ ) are not reproduced by the simplified theoretical treatment. In addition, the stationary probability density function defined in Eqs. (12) and (13) clearly shows, for constant values of $D$ and $\tau$ and as a function of $q$, the transition from unimodal to bimodal behavior. Again, these general results and tendencies, together with the reentrance effect, have been confirmed by extensive numerical simulations as shown in Figs. 1-3. However, the agreement between theory and simulations is only qualitative, as the first are unable to reproduce some of the details revealed by the second. For instance, from the numerical simulations a more complex behavior of the transition line for large $\tau$ is apparent, while it is not present in the theoretical predictions (see Fig. 1) being worth remarking here that they are strictly valid only for $q \approx 1$. 
These results are in the same line that a series of previous studies on noise-induced phenomena where the noise source was non-Gaussian. In all the cases, we have found that the response of the system results to be enhanced or to have a strong dependence for values of the parameter $q$ corresponding to a departure from Gaussian behavior. Some of such studies, including stochastic resonance [22,23], gated traps [25], Brownian motors [26], have been reviewed in [19].

Even though our results are so far only preliminary and further studies are required, we expect that the phenomenon discussed here will have remarkable effects on the noise-induced phase transitions [6] as well as in the problem of coupled ratchets $[9,10]$. For instance, we can expect changes in the phase diagram of the first, or in the hysteresis cycle of the second, that could have interesting consequences in technological applications. Such studies will be the subject of further work.

\section{Acknowledgements}

This works is supported by MCyT (Spain) and FEDER, projects BFM2001-0341-C02-01 and BFM2000-1108. HSW acknowledges the partial support from CONICET and ANPCyT, both Argentine agencies, and thanks the MECyD, Spain, for an award within the Programa de Sabáticos para Profesores Visitantes, and the Universitat de les Illes Balears for the kind hospitality extended to him.

\section{Appendix A. Numerical simulations}

In order to test our theoretical predictions, we have resorted to numerical simulations of the system indicated by Eqs. (8) and (2) and (3). For the numerical integration of those equations we have used a second-order stochastic Runge-Kutta type algorithm [27]. For the general problem

$$
\dot{x}=f(x)+g(x) \eta(t), \quad \dot{\eta}=q(\eta)+\epsilon \xi(t),
$$

where $\xi(t)$ is a white noise of mean equal to zero and correlations $\left\langle\xi(t) \xi\left(t^{\prime}\right)\right\rangle=\delta\left(t-t^{\prime}\right)$, the algorithm is as following: time is discretized in steps of size $h$ and the value of the process $\eta(t)$ at the discretized times is obtained by the recursion relation

$$
k(t)=h q(\eta(t)), \quad l(t)=\epsilon h^{1 / 2} G(t), \quad \eta(t+h)=\eta(t)+\frac{1}{2} h[q(\eta(t))+q(\eta(t)+k(t)+l(t))]+l(t)
$$

with the initial value $\eta(0)=0$. Here the numbers $G(t)$ for different times $t$ are independent Gaussian random numbers of mean equal to zero and variance equal to one. They have been obtained by using a particularly efficient generator [28]. In the case $q<1$, it might occur that the generated value of $G(t)$ is such that $\eta(t+h)$ is outside the cut-off interval $\left(-\eta_{\mathrm{c}}, \eta_{\mathrm{c}}\right)$. If this happens, that value is discarded and a new $G(t)$ is generated until the condition $|\eta(t+h)|<\eta_{\mathrm{c}}$ is fulfilled. We have found that the percentage of discarded $G(t)$ values is less than $5 \times 10^{-4}$ in the worst case analyzed here corresponding to $q=0.5, \tau=4$. No such a problem ever occurs for $q \geq 1$.

Once the values of $\eta(t)$ at the discretized times have been obtained, the integration of (A.1) proceeds with a standard Runge-Kutta second order method:

$$
k_{x}(t)=f(x(t)), \quad x(t+h)=x(t)+\frac{1}{2} h\left[f(x(t))+f\left(x(t)+k_{x}(t)\right)+g(x(t)) \eta(t)+g\left(x(t)+k_{x}(t)\right) \eta(t+h)\right] .
$$


We have used a time step $h=0.01$ and the histogram for $P^{\text {st }}(x)$ has been obtained from $10^{8}$ values of $x$ separated by a time $t=1$ (corresponding, hence, to 100 integration steps).

\section{References}

[1] L. Gammaitoni, P. Hänggi, P. Jung, F. Marchesoni, Rev. Mod. Phys. 70 (1998) 223.

[2] J.F. Lindner, B.K. Meadows, W.L. Ditto, M.E. Inchiosa, A. Bulsara, Phys. Rev. E 53 (1996) 2081.

[3] F. Castelpoggi, H.S. Wio, Europhys. Lett. 38 (1997) 91;

F. Castelpoggi, H.S. Wio, Phys. Rev. E 57 (1998) 5112;

S. Bouzat, H.S. Wio, Phys. Rev. E 59 (1999) 5142;

H.S. Wio, B. Von Haeften, S. Bouzat, Physica A 306C (2002) 140.

[4] W. Horsthemke, R. Lefever, Noise-Induced Transitions: Theory and Applications in Physics, Chemistry and Biology, Springer, Berlin, 1984.

[5] C. Van den Broeck, J.M.R. Parrondo, R. Toral, Phys. Rev. Lett. 73 (1994) 3395;

C. Van den Broeck, J.M.R. Parrondo, R. Toral, R. Kawai, Phys. Rev. E 55 (1997) 4084.

[6] S. Mangioni, R. Deza, H.S. Wio, R. Toral, Phys. Rev. Lett. 79 (1997) 2389;

S. Mangioni, R. Deza, R. Toral, H.S. Wio, Phys. Rev. E 61 (2000) 223.

[7] P. Reimann, Phys. Rep. 361 (2002) 57.

[8] R.D. Astumian, P. Hänggi, Phys. Today 55 (11) (2002) 33.

[9] P. Reimann, R. Kawai, C. Van den Broeck, P. Hänggi, Europhys. Lett. 45 (1999) 545.

[10] S. Mangioni, R. Deza, H.S. Wio, Phys. Rev. E 63 (2001) 041115.

[11] J. García-Ojalvo, J.M. Sancho, Noise in Spatially Extended Systems, Springer-Verlag, New York, 1999.

[12] S.M. Bezrukov, I. Vodyanoy, Nature 378 (1995) 362;

A. Fuliński, Phys. Rev. Lett. 79 (1997) 4926;

I. Goychuk, P. Hänggi, Phys. Rev. E 61 (2000) 4272;

D. Nozaki, D.J. Mar, P. Griegg, J.D. Collins, Phys. Rev. Lett. 72 (1999) 2125;

K. Wiesenfeld, D. Pierson, E. Pantazelou, Ch. Dames, F. Moss, Phys. Rev. Lett. 52 (1994) 2125;

A. Manwani, PhD Thesis, CALTECH, 2000.

[13] P. Hänggi, P. Risenborough, Phys. Rev. A 27 (1983) 3379;

C. Van den Broeck, P. Hänggi, Phys. Rev. A 30 (1984) 2730.

[14] A.V. Soldatov, Mod. Phys. Lett. B 7 (1993) 1253.

[15] F. Castro, A. Sánchez, H.S. Wio, Phys. Rev. Lett. 75 (1995) 1691.

[16] L. Borland, Phys. Lett. A 245 (1998) 67.

[17] C. Tsallis, J. Statist. Phys. 52 (1988) 479;

E.M.F. Curado, C. Tsallis, J. Phys. A 24 (1991) L69;

E.M.F. Curado, C. Tsallis, J. Phys. A 24 (1991) 3187;

E.M.F. Curado, C. Tsallis, J. Phys. A 25 (1992) 1019;

C. Tsallis, Phys. World 10 (1997) 42.

[18] S. Abe, Y. Okamoto (Eds.), Nonextensive Statistical Mechanics and its Applications, Springer, Berlin, 2001; M. Gell-Mann, C. Tsallis (Eds.), Nonextensive Entropy_-Interdisciplinary Applications, Oxford University Press, Oxford, 2003.

[19] H.S. Wio, On the Role of Non-Gaussian Noises in Noise Induced Phenomena, Chapter in Ref. [18].

[20] M.A. Fuentes, H.S. Wio, R. Toral, Physica A 303 (2002) 91.

[21] P. Jung, P. Hänggi, Phys. Rev. A 35 (1987) 4464;

L. H'walisz, P. Jung, P. Hänggi, P. Talkner, L. Schimansky-Geier, Z. Phys. B 77 (1989) 471; P. Hänggi, Chem. Phys. 180 (1994) 157.

[22] M.A. Fuentes, R. Toral, H.S. Wio, Physica A 295 (2001) 114.

[23] F.J. Castro, M.N. Kuperman, M.A. Fuentes, H.S. Wio, Phys. Rev. E 64 (2001) 051105.

[24] P. Colet, H.S. Wio, M. San Miguel, Phys. Rev. A 39 (1989) 6094;

H.S. Wio, P. Colet, L. Pesquera, M.A. Rodriguez, M. Sa Miguel, Phys. Rev. A 40 (1989) 7312;

H.S. Wio, Application of path integration to stochastic processes: an introduction, in: G. Zgrablich (Ed.), Fundamentals and Applications of Complex Systems, Nueva Ed. Univ., U.N. San Luis, 1999, p. 253.

[25] A.D. Sánchez, J.A. Revelli, H.S. Wio, Phys. Lett. A 277 (2000) 304;

H.S. Wio, J.A. Revelli, A.D. Sánchez, Physica D 168-169 (2002) 165.

[26] S. Bouzat, H.S. Wio, Strong enhancement of current, efficiency and mass separation in Brownian motors driven by non-Gaussian noises. cond-mat/0112304.

[27] M. San Miguel, R. Toral, Stochastic effects in physical systems, in: E. Tirapegui, J. Martínez, R. Tiemann (Eds.), Instabilities and Nonequilibrium Structures VI, Kluwer Academic Publishers, Dordrecht, 1999, pp. 35-130.

[28] R. Toral, A. Chakrabarti, Comput. Phys. Commun. 74 (1993) 327. 